\title{
Épocas críticas de competencia de arvenses en cultivo de yuca en el Caribe seco colombiano
}

\section{Critical times for weed competition on cassava cultivation in the colombian dry Caribbean}

\author{
José Antonio Rubiano-Rodríguez ${ }^{1 *}{ }^{\oplus}$; Carina Cecilia Cordero-Cordero ${ }^{2 \oplus}$ \\ Recibido para publicación: Marzo 1 de 2019 - Aceptado para publicación: Mayo 24 de 2019
}

\begin{abstract}
RESUMEN
La yuca (Manihot esculenta Crantz) pertenece a la familia Euphorbiaceae, su centro de origen genético se encuentra en la Cuenca Amazónica y es uno de los cultivos que produce la mayor cantidad de energía, siendo una de las raíces más comercializadas a nivel mundial, principalmente como fuente de carbohidratos. Sin embargo, el cultivo se puede ver afectado por problemas fitosanitarios como los insectos, hongos y malas hiervas o arvenses entre otros. Las arvenses pueden ser el mayor problema fitosanitario en los cultivos comerciales. Existen reportes que debido al no manejo de las arvenses en el cultivo de yuca las pérdidas pueden llegar a ser drásticas y alcanzar hasta un $50 \%$ en la producción final. El objetivo de este trabajo fue evaluar las épocas críticas de competencia de malezas sobre el cultivo de yuca. Se evaluaron 10 tratamientos con tres repeticiones, se tomaron datos mensuales del porcentaje de cobertura, la altura de las malezas y diferentes variables al momento de la cosecha incluyendo rendimiento. El peso fresco y contenido de materia seca de las malezas tiene tendencia aumentar principalmente en los tratamientos en los cuales permanecen por mayor periodo de tiempo. La época donde las malezas pueden llegar a ser más críticas e influir en los rendimientos del cultivo de yuca está entre los 60 y 90 días después de siembra.
\end{abstract}

Palabras clave: Malezas; Control; Yuca; Cobertura; Competencia; Época.

\footnotetext{
'Corporación Colombiana de Investigación Agropecuaria AGROSAVIA, Centro de Investigación La Selva.

${ }^{2}$ Corporación Colombiana de Investigación Agropecuaria AGROSAVIA, Centro de Investigación Motilonia.

*Autor para correspondecia: Carina Cordero

Email: ccordero@agrosavia.co
}

\begin{abstract}
Cassava (Manihot esculenta Crantz) is one of the crops that produces the greatest amount of energy, mainly as a source of carbohydrates. However, the crop can be affected by phytosanitary problems such as insects, fungi and weeds, among others. Weeds can be the worst sanitary problem in commercial crops. Reports of wrong weed management in cassava crops can reduce up to $50 \%$ final production. The objective of this work was to evaluate the critical period of weed competition in cassava cultivation. Ten treatments, with three replicates each, were evaluated. Weed percentage coverage, weed fresh weight, weed dry matter content, weed height, plant height, number of plants harvested, dry matter percentage, crop index, yield, per plot and per hectare, were evaluated. Weed fresh weight and dry matter content tend to increase when remaining for longer period of time (179.3 and $114.5 \mathrm{gr}$ for T1 and T9 respectively for fresh weight and $36.4 \mathrm{gr}$ for dry matter in T6). The largest weed coverage in the crop affected the height of the cassava plants and the number of plants harvested. Critical time for weeds influencing yield of cassava cultivation is between 60 and 90 days after planting.
\end{abstract}

Key words: Weeds; Control; Cassava; Coverage; Competition; Season. crear a partir del material, de forma no comercial, dando crédito y licencia de forma adecuada a los autores de la obra. 


\section{INTRODUCCIÓN}

La yuca Manihot esculenta Crantz pertenece a la familia Euphorbiaceae, su centro de origen genético se encuentra en la Cuenca Amazónica. Se han descrito alrededor de 98 especies del género Manihot de las cuales sólo la yuca tiene relevancia económica y es cultivada (Aristizabal et al., 2007). La planta de yuca se cultiva en diversas condiciones tropicales, su ventaja comparativa con otros cultivos más rentables es su capacidad para producir en suelos ácidos, de escasa fertilidad, con precipitaciones esporádicas o largos períodos de sequía (Aristizabal et al., 2007). La yuca, es uno de los cultivos que produce la mayor cantidad de energía. Siendo una de las raíces más comercializadas a nivel mundial, principalmente como fuente de carbohidratos (Rojas et al., 2007). Sin embargo, el cultivo se puede ver afectado por problemas fitosanitarios como los insectos, hongos y malas hiervas o arvenses entre otros. Según Calle (2002), las arvenses son el mayor problema fitosanitario en los cultivos comerciales.

Existen reportes que debido al no manejo de las arvenses las pérdidas pueden llegar a ser drásticas y alcanzar hasta un $50 \%$ en la producción final (CIAT 1981; Báez et al., 1998). Según Aristizabal et al. (2007), para evitar este nivel de pérdidas se debe realizar el control de malezas durante los primeros 60 días buscando evitar el crecimiento de gramíneas y plantas de hoja ancha que compitan por luz, agua y nutrientes. Este control puede realizarse de forma manual, mecánica o química. Según Barreyro y Sánchez (2002), el manejo de malezas es una herramienta de valor para incrementar los rendimientos, haciendo mejor aprovechamiento de los recursos. Esto implica conocer los factores que interactúan al establecerse la competencia, tales como el período de mayor susceptibilidad (Girón y Alfonso, 2000), la cobertura de las malezas, la dinámica de crecimiento del cultivo y la magnitud de las pérdidas de rendimiento registradas (Quiñónez y Moreno 1995). La determinación del período crítico de competencia es un dato propio de cada ambiente agroecológico que permite la planificación del manejo de malezas en el cultivo (Aldrich y Kremer, 1997; Radosevich et al., 1997). Sin embargo, son muy escasos los estudios sobre el control de malezas en el cultivo de yuca, ya sea manual o químico (Gutiérrez et al., 2008). Además, en la zona agroecológica Caribe Seco Colombiano, no se ha delimitado el período crítico de competencia en yuca. Para tratar de dilucidar estos interrogantes se realizó un ensayo en el $\mathrm{Cl}$ Motilionia, teniendo como objetivo principal la evaluación de épocas críticas y manejo adecuado de las malezas.

\section{MATERIALES Y MÉTODOS}

El estudio se realizó en lote número seis del Cl Motilonia 1000`2.4" N; 73¹5’21,3” O, municipio de Agustín Codazzi - Cesar, en el periodo julio de 2016 a mayo de 2017.

Se efectuó un pase de rastra y dos pases de rastrillo pulidor, se formaron camellones de 0,3 metros de altura y 1,0 metro de distancia entre centros de los surcos.

Se seleccionó semilla de la variedad Venezolana, las cuales se cortaron en esquejes de 20 a $25 \mathrm{~cm}$ de longitud, procedentes de parcelas de agricultores de La Guajira. Antes de la siembra la semilla fue desinfectada en una mezcla de agua ( $100 \mathrm{~L}$ ) con 0,4 L de Cipermetrina y $2 \mathrm{~kg}$ Mancozeb, sumergiendo las estacas durante 15 minutos en la mezcla. Posteriormente se realizó la siembra con una distancia de 1,0 metro entre surcos y 1,0 metro entre plantas. Las estacas se insertaron en el suelo hasta la mitad de su longitud y en ángulo recto.

Se realizó un diseño de bloques completos al azar con 10 tratamientos y tres repeticiones 
por tratamiento, siendo los tratamientos: $\mathrm{T} 1$ = Sin control todo el ciclo del cultivo, $\mathrm{T} 2=$ Limpio todo el ciclo, T3 = Sin control hasta los 30 días después de la siembra (dds), T4 = Con control hasta los 30 dds, T5 = Sin control hasta los 60 dds, T6 = Con control hasta los 60 dds, $\mathrm{T} 7=$ Sin control hasta los $90 \mathrm{dds}, \mathrm{T} 8=$ Con control hasta los 90 dds, T9 = Sin control hasta los 120 dds, $\mathrm{T} 10=$ Con control hasta los 120 dds.

La unidad experimental fue de cinco metros de ancho x 10 metros de largo, para un área de $50 \mathrm{~m}^{2}$, teniendo cinco surcos por repetición en cada tratamiento y un total de 50 plantas.

Durante el desarrollo del cultivo se evaluó mensualmente la cobertura de malezas presentes en los tratamientos respectivos en cada fecha. Para lo cual, se lanzó un marco de $25 \mathrm{~cm} \times 25 \mathrm{~cm}$ tres veces por parcela aleatoriamente. Se registró el porcentaje de área ocupada por las malezas en cada lanzamiento y se clasificaron (Hoja ancha, gramíneas y otras). Además, se evaluaron variables como, altura de malezas, peso fresco total de malezas por cuadro, peso fresco separado (Hoja ancha, gramíneas y otras). Para determinar el peso seco, se tomó una muestra de $300 \mathrm{gr}$ del peso fresco total y se secaron en estufa $80^{\circ} \mathrm{C}$ durante 48 horas. El porcentaje de materia seca (\% MS) de las malezas se obtuvo aplicando la ecuación 1.

$$
\% M S=100-\frac{p f-p s}{p f}-100
$$

Donde: $p f=p e s o$ fresco de la muestra; $p s=p e s o$ seco de la muestra

Al momento de la cosecha de las plantas de yuca se evaluaron otras variables tales como: Altura de planta, diámetro del tallo a $80 \mathrm{~cm}$ del suelo, número de plantas cosechadas por parcela, numero de raíces comerciales, peso de raíces comerciales, numero de raíces no comerciales, peso de raíces no comerciales, peso total de raíces, numero de semillas/planta, peso total aéreo, peso raices frescas en aire, peso raíces frescas en agua, porcentaje de materia seca, índice de cosecha, producción por parcela y rendimiento. Para esta evaluación de seleccionaron cinco plantas por parcela o repetición de cada uno de los tratamientos. Para determinar el peso fresco tanto en agua como aire, se tomó una muestra de $3 \mathrm{~kg}$ por repetición en cada tratamiento. El porcentaje del contenido de materia seca en las raíces y el índice de cosecha se determinaron mediante las ecuaciones 2 y 3 respectivamente.

$$
\% M S=\left[158,3 \times\left(\frac{p f \cdot a r}{\text { pf.ar-pf.ag }}\right)-142\right]
$$

Donde: $p f . a r=$ Peso fresco en aire; pf.ag $=$ Peso fresco en agua.

$$
\mathrm{IC}=\left(\frac{P T R}{P T A+P T R}\right)
$$

Donde: $P T R=$ Peso total de raíces; $P T A=$ Peso total aéreo.

Se realizó análisis de varianza y pruebas de separación de medias (Tukey, 0.05\%) cuando fue necesario con el programa estadístico SAS 9.3 (SAS 2011). Además, se hizo una correlación de Pearson entre el número de plantas cosechadas por parcela y la producción de raíces.

\section{RESULTADOS Y DISCUSIÓN}

De acuerdo con lo mostrado en la Figura 1, aquellos tratamientos (T1, T4, T7 y T9), los cuales permanecen bajo la presión de malezas al menos 60 días, la cobertura de estas fue superior al 90\%. Este comportamiento no coincide con lo reportado por Arismendi (2001), quien afirma que para las condiciones de las sabanas de Monagas y Anzoategui en Venezuela, hay mayor presión de malezas hasta los 120 después de la siembra, periodo crítico de competencia de las mismas en el cultivo. Se presentan algunas diferencias significativas entre los tratamientos en algunos 
de los periodos evaluados, principalmente entre T1 y los tratamientos donde se hace control durante un periodo de la etapa del cultivo correspondientes a T6 (Con control hasta los 60 dds), T7 (Sin control hasta los 90 dds) y T8 (Con control hasta los $90 \mathrm{dds}$ ).

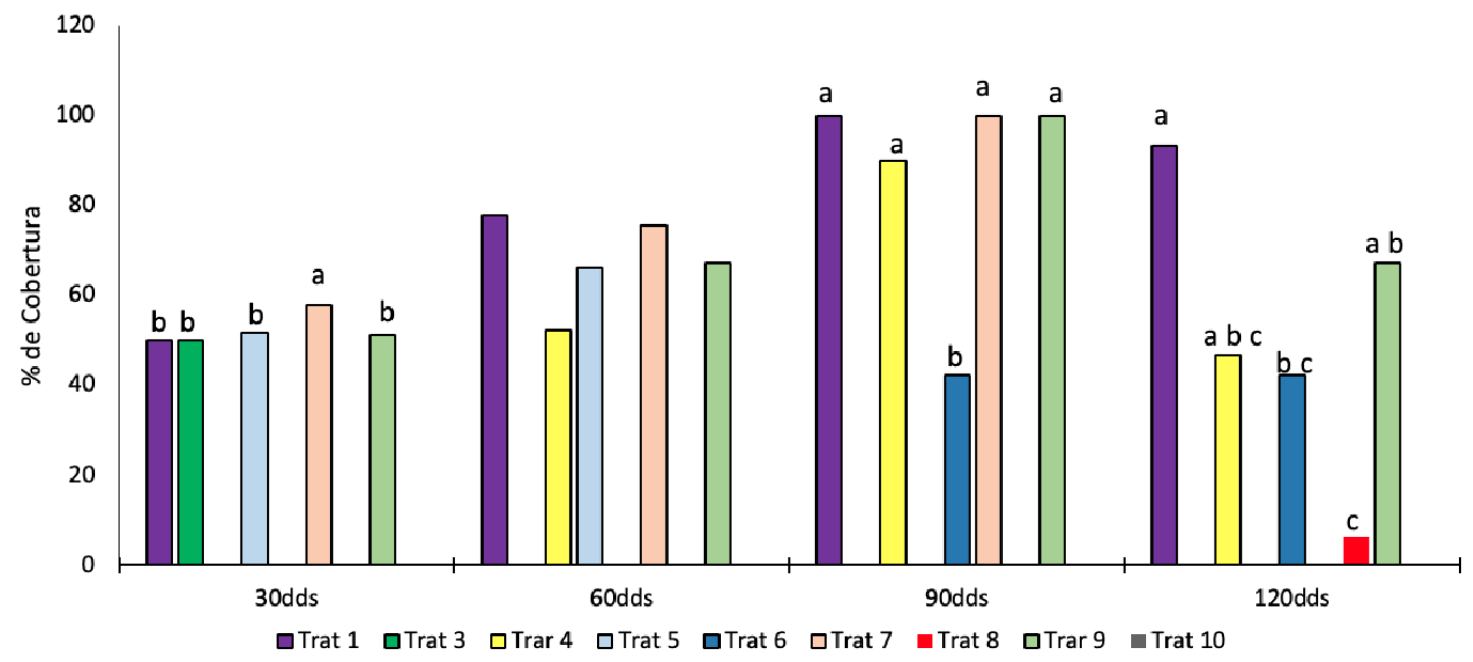

Figura 1. Porcentaje de cobertura de las malezas en cada tratamiento durante los diferentes tiempos de evaluación. Siendo Trat1 (Sin control todo el ciclo del cultivo); Trat3 (Sin control hasta los 30 días después de la siembra dds); Trat4 (Con control hasta los 30 dds); Trat5 (Sin control hasta los 60 dds); Trat6 (Con control hasta los 60 dds); Trat7 (Sin control hasta los 90 dds); Trat8 (Con control hasta los 90 dds); Trat9 (Sin control hasta los 120 dds); Trat10 (Con control hasta los 120 dds). Barras con igual letra en el mismo tiempo de evaluación no difieren estadísticamente $(\mathrm{P}<0,05)$.

Se presentaron diferencias estadísticas de la altura de malezas entre los tratamientos a los 60, 90 y 120 dds, principalmente T8 vs los tratamientos donde no se venía haciendo control a los 90 dds, periodo en el cual las malezasllegaronsupuntomáximodecrecimiento (Figura 2). Lo anterior indica que las malezas presentaron mayor altura cuando el cultivo estuvo sin control de las mismas durante todo el ciclo (T1) y cuando el cultivo estuvo sin control de malezas hasta los 120 dds (T9).

Los resultados para las variables cobertura de las malezas y altura de planta de las malezas en los tratamientos con control de las mismas, se puede atribuir a una menor competencia de las malezas con el cultivo, lo que permitió un rápido cierre de calles por el crecimiento del follaje de las plantas de yuca. Según Calle (2000), las arvenses en el cultivo de yuca pueden permanecer y desarrollarse hasta que haya un traslape del follaje del cultivo, que generalmente ocurre entre los 90 y 120 dds. Con lo anterior se puede inferir que los primeros 30 a 60 dds, se debe hacer control de arvenses tal como lo recomiendan en algunas investigaciones (CIAT, 1981).

De acuerdo con lo reportado por Mejía de Tafur (1994), durante los primeros 3 meses del cultivo de yuca, la formación de hojas tiene prioridad sobre la formación de raíces de almacenamiento; después la planta continúa formando hojas a la vez que almacena almidón en las raíces. Por su parte, el índice de área foliar aumenta los 3 y 6 meses de edad del cultivo. Por lo anterior, y de acuerdo a lo reportado por el Instituto Nacional de Innovación Agraria (2014), el control de malezas en el cultivo de yuca debe empezar tan pronto como aparezcan las malas hierbas; es importante realizarlo durante los primeros meses, después de la siembra, ya que las plantas jóvenes son más susceptibles a la competencia con las malezas, por luz, agua y nutrientes.

El peso fresco (Tabla 1) y contenido de materia seca de las malezas (Tabla 2) tiende a aumentar principalmente en los tratamientos en los cuales permanecen por mayor periodo 


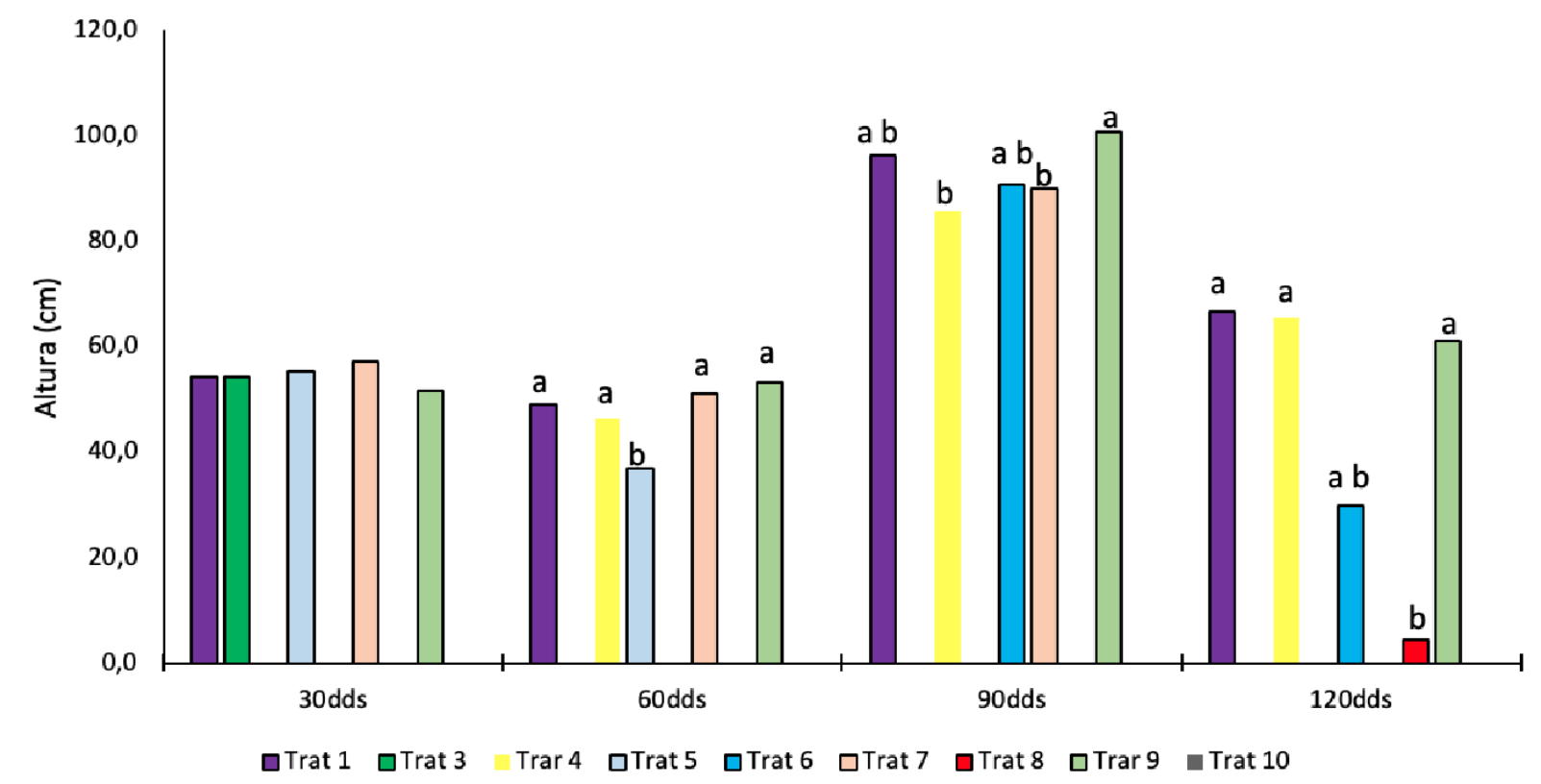

Figura 2. Altura promedio de las malezas en cada tratamiento durante los diferentes tiempos de evaluación, Siendo Trat1 (Sin control todo el ciclo del cultivo); Trat3 (Sin control hasta los 30 días después de la siembra dds); Trat4 (Con control hasta los $30 \mathrm{dds}$ ); Trat5 (Sin control hasta los $60 \mathrm{dds}$ ); Trat6 (Con control hasta los $60 \mathrm{dds}$ ); Trat7 (Sin control hasta los 90 dds); Trat8 (Con control hasta los 90 dds); Trat9 (Sin control hasta los 120 dds); Trat10 (Con control hasta los 120 dds).. Barras con igual letra en el mismo tiempo de evaluación no difieren estadísticamente $(\mathrm{P}<0,05)$.

de tiempo en el sistema productivo de yuca. El peso fresco (Tabla 1) y contenido de materia seca de las malezas (Tabla 2) tiende a aumentar principalmente en los tratamientos en los cuales permanecen por mayor periodo de tiempo en el sistema productivo de yuca. Este resultado coincide con lo reportado por Blanco et al. (2014), quienes al evaluar el periodo crítico de competencia de arvenses en el cultivo de maíz, encontraron que la producción de biomasa de las arvenses fue mayor en los tratamientos donde permanecieron las arvenses la mayor cantidad de tiempo.

El peso fresco presentó los valores más altos a los 60 y 90 dds (en todos los tratamientos, a excepción de los tratamientos T1 y T6, en donde se obtuvieron valores altos para esta variable a los $120 \mathrm{dds}$ ), presentando diferencias significativas entre los tratamientos (Tabla 1). En el caso del contenido de materia seca de las malezas, el valor más alto se presentó a los 90 dds, pero con diferencias significativas entre los tratamientos a los 60 dds (Tabla 2).
En los tratamientos que permanecieron por más tiempo con presencia de malezas, los valores de altura de las plantas fueron más bajos en comparación con los tratamientos donde se hizo manejo de las mismas. Las plantas de T1 y T7 son estadísticamente más bajas que las de T10 (Tabla 3). Lo que indica que cuando el cultivo estuvo sin control todo el ciclo y sin control hasta los 90 días después de la siembra, por el alto porcentaje de cobertura de malezas (Figura 1), éstas fueron más competitivas que las plantas de yuca, por los recursos agua, luz y nutrientes.

Este comportamiento coincide con lo reportado por Hruska (1987), quien, al evaluar el efecto de la cobertura de suelo por las malezas en dos variedades de yuca, encontró que las plantas cultivadas en áreas enmalezadas sufrieron mayor atrofiamiento que las cultivadas en áreas menos enmalezadas. De acuerdo a lo reportado por Pérez y Ruiz (2010), el crecimiento del cultivo de yuca es lento en los primeros meses, por lo tanto, el control de malezas es esencial para un correcto desarrollo. 
Rubiano-Rodíguez; Cordero-Cordero - Competencia de malezas en yuca.

Tabla 1. Valores promedios del peso fresco de las malezas en los diferentes tiempos de evaluación.

\begin{tabular}{ccccc}
\hline Tratamientos & 30dds & $\mathbf{6 0 d d s}$ & $\mathbf{9 0 d d s}$ & $\mathbf{1 2 0 d d s}$ \\
\hline T1 & 77,03 & $131,4 \mathrm{AB}$ & $113,3 \mathrm{~A}$ & $179,3 \mathrm{~A}$ \\
T3 & 61,77 & - & - & - \\
T4 & - & $117,7 \mathrm{AB}$ & $81,2 \mathrm{AB}$ & $57,3 \mathrm{AB}$ \\
T5 & 73,27 & $212,3 \mathrm{~A}$ & - & - \\
T6 & - & - & $27,3 \mathrm{~B}$ & $54,3 \mathrm{AB}$ \\
T7 & 69,73 & $130 \mathrm{AB}$ & $115,4 \mathrm{~A}$ & - \\
T8 & - & - & - & $3,8 \mathrm{~B}$ \\
T9 & 57,67 & $140,3 \mathrm{AB}$ & $109 \mathrm{~A}$ & $114,5 \mathrm{AB}$ \\
T10 & - & - & - & - \\
CV & $\mathbf{2 9 , 0 3}$ & $\mathbf{3 0 , 9 1}$ & $\mathbf{4 3 , 5 1}$ & $\mathbf{5 9 , 7 7}$ \\
\hline
\end{tabular}

Siendo T1 (Sin control todo el ciclo del cultivo); T2 (Limpio todo el ciclo); T3 (Sin control hasta los 30 dds); T4 (Con control hasta los 30 dds); T5 (Sin control hasta los 60 dds); T6 (Con control hasta los 60 dds); T7 (Sin control hasta los 90 dds); T8 (Con control hasta los 90 dds); T9 (Sin control hasta los 120 dds); T10 (Con control hasta los 120 dds). Columnas con igual letra no difieren estadísticamente según la prueba de Tukey. $(\mathrm{P}<0.05)$. dds= días después de siembra. $(-)$ No hay dato.

Para la variable número de plantas cosechadas, se presentaron diferencias estadísticas entre los tratamientos (Tabla 3). En los tratamientos en los cuales hubo presión de arvenses sobre el cultivo por más de 90 días se observó un menor número de plantas cosechadas, correspondientes a T1 (8 plantas), T7 (12 plantas) y T9 (26 plantas). En el trabajo realizado por Paredes (2014), se encontró para el cultivo de yuca una gran diferencia de producción entre las parcelas limpias todo el tiempo y las parcelas con malezas todo el tiempo.

Tabla 2. Valores promedios del porcentaje de materia seca de las malezas en los diferentes tiempos evaluados

\begin{tabular}{ccccc}
\hline Tratamientos & 30dds & 60dds & 90dds & 120dds \\
\hline T1 & 27,9 & $30,2 \mathrm{~A}$ & 31,7 & 26,2 \\
T3 & 32,1 & - & - & - \\
T4 & - & $20,3 \mathrm{~B}$ & 29,4 & 36,4 \\
T5 & 24,9 & $22,1 \mathrm{~B}$ & - & - \\
T6 & - & - & 36,4 & 24,8 \\
T7 & 27,2 & $26,6 \mathrm{AB}$ & 28,8 & - \\
T8 & - & - & - & 14,7 \\
T9 & 27,3 & $26,7 \mathrm{AB}$ & 30,6 & 28,4 \\
T10 & - & - & - & - \\
CV & $\mathbf{1 5 , 1 9}$ & $\mathbf{1 2 , 7 3}$ & $\mathbf{1 8 , 3 7}$ & $\mathbf{5 4 , 1 7}$ \\
\hline
\end{tabular}

Siendo T1 (Sin control todo el ciclo del cultivo); T2 (Limpio todo el ciclo); T3 (Sin control hasta los 30 dds); T4 (Con control hasta los 30 dds); T5 (Sin control hasta los 60 dds); T6 (Con control hasta los 60 dds); T7 (Sin control hasta los 90 dds); T8 (Con control hasta los 90 dds); T9 (Sin control hasta los 120 dds); T10 (Con control hasta los 120 dds). Columnas con igual letra no difieren estadísticamente según la prueba de Tukey. $(\mathrm{P}<0.05)$. Tukey. 
Rubiano-Rodíguez; Cordero-Cordero - Competencia de malezas en yuca.

Tabla 3. Valores promedio de las variables fisiológicas evaluadas en yuca al momento de cosecha en cada tratamiento

\begin{tabular}{|c|c|c|c|c|c|c|c|c|c|c|c|}
\hline \multirow{2}{*}{ Variables } & \multicolumn{10}{|c|}{ Tratamientos } & \multirow{2}{*}{$\mathrm{CV}$} \\
\hline & 1 & 2 & 3 & 4 & 5 & 6 & 7 & 8 & 9 & 10 & \\
\hline Altura & $179,7 \mathrm{C}$ & $213,3 \mathrm{BC}$ & $227,7 \mathrm{ABC}$ & $250 \mathrm{AB}$ & $211,67 B C$ & $237,7 \mathrm{ABC}$ & 183,7 C & $263,3 \mathrm{AB}$ & 206,3 BC & $286,0 \mathrm{~A}$ & 13,7 \\
\hline Pta & 5,9 & 8,4 & 6,7 & 8,7 & 9,3 & 7,9 & 7,4 & 9,4 & 7,1 & 6,9 & 23,4 \\
\hline Rc & 3,9 & 3,3 & 1,7 & 2,7 & 4 & 2,9 & 3,5 & 3,6 & 4,1 & 2 & 46,4 \\
\hline Prc & 1,7 & 2 & 1,1 & 1,6 & 2,2 & 1,3 & 2,1 & 1,9 & 2,1 & 0,8 & 58,2 \\
\hline Rnc & 6 & 7,3 & 7,7 & 7,1 & 6,5 & 6,9 & 6,3 & 10 & 6,3 & 7,9 & 38,3 \\
\hline Prnc & 0,9 & 1 & 1,3 & 0,9 & 1,1 & 0,9 & 1 & 1,3 & 1 & 1,1 & 34,8 \\
\hline Ptr & 2,66 & 3,5 & 2 & 2,5 & 3,4 & 2,2 & 3,1 & 3,2 & 3,2 & 1,9 & 32,5 \\
\hline Plcos & $8 \mathrm{D}$ & $39 A B$ & $46 \mathrm{~A}$ & $40,3 \mathrm{AB}$ & $29,7 \mathrm{AB}$ & $32,7 \mathrm{AB}$ & $11,7 \mathrm{CD}$ & $44 \mathrm{~A}$ & $26 \mathrm{BC}$ & $43,3 \mathrm{~A}$ & 27 \\
\hline Renpar & $6,0 \mathrm{E}$ & $95,0 \mathrm{ABC}$ & $97,3 \mathrm{ABC}$ & $105,0 \mathrm{AB}$ & 72,8 BCD & $101,0 \mathrm{ABC}$ & $24,2 \mathrm{DE}$ & $130,3 \mathrm{~A}$ & 49,4 CDE & $138,9 \mathrm{~A}$ & 34,6 \\
\hline Sem & 8,8 & 30,1 & 15,1 & 17,7 & 19,9 & 16 & 23,4 & 24,7 & 11,6 & 17 & 59,7 \\
\hline Dia & 26,3 & 27,4 & 25 & 29,7 & 30,4 & 28,6 & 28,2 & 29,4 & 28,2 & 28,2 & 11,4 \\
\hline MS & $42,3 \mathrm{AB}$ & $39,0 \mathrm{~B}$ & $39,2 A B$ & $41,9 \mathrm{AB}$ & $39,6 \mathrm{AB}$ & $39,1 \mathrm{AB}$ & $39,3 \mathrm{AB}$ & $42,8 \mathrm{~A}$ & $39,2 \mathrm{AB}$ & $41,5 \mathrm{AB}$ & 4,6 \\
\hline IC & $0,46 \mathrm{~A}$ & $0,36 \mathrm{AB}$ & $0,36 \mathrm{AB}$ & $0,29 B$ & $0,38 \mathrm{AB}$ & $0,28 \mathrm{~B}$ & $0,43 \mathrm{AB}$ & $0,33 \mathrm{AB}$ & $0,45 \mathrm{~A}$ & $0,28 \mathrm{~B}$ & 23,2 \\
\hline Rend & $6,1 \mathrm{~B}$ & $24,2 \mathrm{~A}$ & $21,1 \mathrm{~A}$ & $26,6 \mathrm{~A}$ & $24,9 \mathrm{~A}$ & $29,9 \mathrm{~A}$ & $20,9 \mathrm{~A}$ & $30,4 \mathrm{~A}$ & $20,3 \mathrm{~A}$ & $32,06 \mathrm{~A}$ & 29,6 \\
\hline
\end{tabular}

Altura: altura de plantas (cms), Pta: peso total aéreo $(\mathrm{kg})$, Rc: Número de raíces comerciales, Prc: peso de raíces comerciales (kg), Rnc: Número de raíces no comerciales, Prnc: Peso de raíces no comerciales (kg), Ptr: peso total de raíces, Plcos: número de plantas cosechadas, Renpar: rendimiento por parcela (kg pa-1 ${ }^{1}$, Sem: número de semilla, Dia: diámetro, MS: Materia seca, IC: Índice de cosecha, Rend: rendimiento (t ha- ${ }^{1}$ ). Fuente propia.

Para la variable \% de MS, T8 fue significativamente mayor a T2, con valores de 42,78 y $39,05 \%$ respectivamente. El T1 (42,35\%) comportó estadísticamente similar al T8 $(42,78$ $\% 9$. En todos los tratamientos evaluados el \% de materia seca fue superior al 35\% (Tabla 3). Los 3 primeros meses del cultivo son críticos para la acumulación de almidón en las raíces de la planta. Según Mejía de Tafur (1994), a los 2 ó 3 meses después de la siembra, algunas de las raíces fibrosas comienzan a acumular almidón, proceso que continúa hasta la cosecha final.
El índice de cosecha (IC) presentó diferencias estadísticas entre tratamientos, destacándose los valores de T1 y T9 (0,46 y $0,45)$, siendo superiores a los valores de T4, T6 y T10 $(0,29 ; 0,28$ y 0,28), los cuales son los más bajos que se presentaron (Tabla 3). El comportamiento observado en las plantas de T1 y T9 las cuales tuvieron mayor tiempo de presión de malezas, sepuedeatribuir a una mayor traslocación y redistribución de almidones hacia las raíces por parte de la planta, para tratar de sobrevivir ante esta condición de 
estrés. Además, es posible que la conservación de humedad en el suelo por la presencia de malezas haya incidido en este comportamiento. Al respecto, Cadavid et al. (1993), encontraron que al emplear mulch de malezas, se favoreció la retención de agua en un suelo arenoso de la Costa Atlántica, y se observó un incremento en los rendimientos del cultivo de yuca.

Aunque hubo diferencias estadísticas en el porcentaje de materia seca de las raíces de yuca entre los tratamientos, todos los valores están en un nivel deseado el cual debe ser superior al 35\% (Marín et al., 2008). De igual forma los valores del índice de cosecha encontrado en este estudio están en el rango de un buen material, en la mayoría de los tratamientos y concuerdan con los reportados por diferentes investigadores (Ceballos et al., 2002; Caraballo y Velásquez, 2000)

Para la variable rendimiento por parcela, los resultados indican que los tratamientos donde no se controló malezas durante los primeros 120 dds, el rendimiento fue más bajo. Los tratamientos corresponden a T1, T7 y T9 en los cuales la producción fue estadísticamente inferior a T4, T8 y T10 (Tabla 3), que corresponden a tratamientos en los cuales se hizo control de malezas desde los 30 hasta los 120 días después de la siembra. Se presentó disminución en el rendimiento desde un 30\% en T3 hasta más de un 90\% en T1. (Figura 3).

En las plantas pertenecientes a los tratamientos (T1, T5, T7 y T9), los cuales permanecieron sin control de malezas por lo menos 60 días del ciclo del cultivo, se pudo haber afectado el normal crecimiento y desarrollo de formación de sus raíces fibrosas. Esto se vio reflejado en la producción por parcela. Mejía de Tafur (1994) indica que durante las primeras semanas del cultivo, la planta de yuca forma raíces fibrosas.

El comportamiento de la variable rendimiento por hectárea (t.ha- ${ }^{1}$ ) fue similar al observado para la variable rendimiento por parcela. Los resultados indican que los tratamientos donde no se controló malezas durante los primeros 120 dds, el rendimiento fue menor. Se puede inferir que dicho efecto se debe más a la disminución del número de plantas cosechadas en promedio por tratamiento debido a la presión

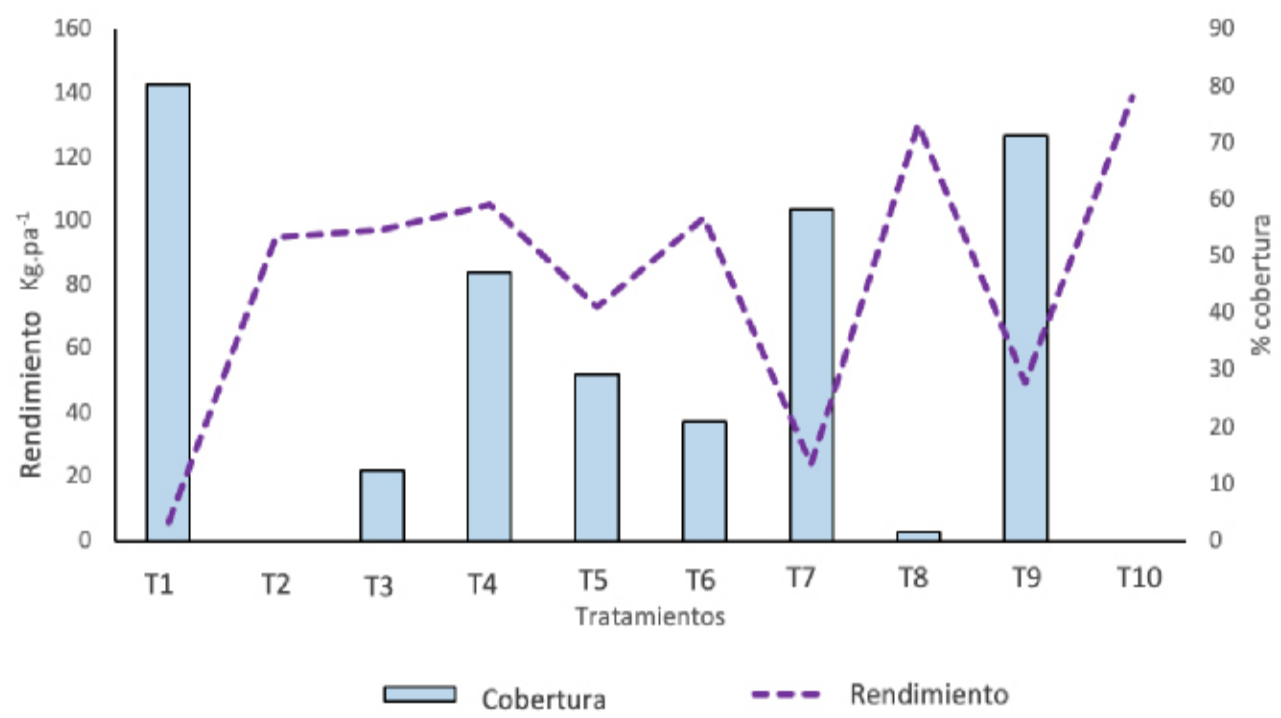

Figura 3. Relación del rendimiento/parcela con el porcentaje de cobertura de las malezas en cada uno de los tratamientos. Siendo T1 (Sin control todo el ciclo del cultivo); T2 (Limpio todo el ciclo); T3 (Sin control hasta los 30 días después de la siembra dds); T4 (Con control hasta los 30 dds); T5 (Sin control hasta los 60 dds); T6 (Con control hasta los 60 dds); T7 (Sin control hasta los 90 dds); T8 (Con control hasta los 90 dds); T9 (Sin control hasta los 120 dds); T10 (Con control hasta los 120 dds). 
de las malezas, que a los resultados obtenidos para la variable índice de cosecha.

Los resultados de este estudio para los tratamientos que estuvieron bajo la presión de arvenses durante los primeros 60-90 días del cultivo, ratifican algunos reportes donde indican que el periodo crítico de las malezas en el cultivo de yuca son los primeros $60 \mathrm{dds}$ (CIAT, 1981). Estudios similares realizados en República Dominicana concuerdan con los aquí obtenidos, donde indican que el tratamiento que permaneció todo el ciclo enmalezado fue el del rendimiento más bajo (IDIAF, 2001).

Blanco et al. (2014), al evaluar el periodo crítico de competencia de arvenses en el cultivo de maíz, encontraron que los rendimientos de este cultivo fueron mayores cuando el cultivo fue atendido en su período crítico alcanzándose valores entre 11 y 12 t.ha- ${ }^{1}$, mientras que los menores rendimientos ( 6 y 7 t.ha- ${ }^{1}$ ) coincidieron con los tratamientos de manejo hasta los 24 días y enmalezado hasta los 48 días.

Por su parte, Paredes (2014) al determinar el período crítico de control de malezas en yuca sembrada a dos densidades en la Provincia de Orellana, encontró que para la densidad de 10.000 plantas por hectárea, este periodo correspondió desde el día 44 al día 123 DDS, indicando, que en estos primeros días se debe controlar la maleza para obtener los mejores rendimientos de producción de este cultivo.

De acuerdo a el análisis de correlación de Pearson, se obtuvo una correlación positiva y altamente significativa (Prob $>|r|$ suponiendo $\mathrm{HO}$ : $\mathrm{Rho}=0$ ) del rendimiento por parcela vs el número de plantas cosechadas, cuyo coeficiente correspondió a 0,86. De acuerdo con la regresión lineal $\left(\mathrm{R}^{2}\right.$ de 0,74$)$, existe una relación directa entre el número total de plantas cosechas y el rendimiento de raíces en cada tratamiento.

\section{CONCLUSIONES}

El peso fresco y contenido de materia seca de las malezas tiene tendencia a aumentar cuando éstas permanecen por mayor periodo de tiempo en el cultivo. Igualmente, la mayor cobertura de malezas en el cultivo afecta la altura de las plantasyelnúmerodeplantasdeyucacosechadas.

A pesar de que a mayor presión de malezas se presenta disminución del contenido de materia seca en las raíces de la planta de yuca, se observa que las plantas en las parcelas que permanecen por mayor tiempo expuestas a presión de las malezas, muestran como mecanismo de tolerancia ante esta situación de estrés, un aumento en su eficiencia en la traslocación de asimilados hacia este órgano de interés, aprovechando la humedad en la zona radicular proporcionada por la cobertura de las malezas, lo que se vió reflejado en sus índices de cosecha.

La época más crítica donde las malezas compiten con el cultivo está entre los 60 y 90 días después de la siembra, influyendo en los rendimientos de este.

\section{Conflicto de Intereses}

Los autores declaran que es un trabajo orginal y no existío conflicto de intereses de ningún tipo en la elaboración y publicación del manuscrito.

\section{AGRADECIMIENTOS}

Los autores expresan sus agradecimientos al Ministerio de Agricultura el cual financiación del proyecto "Un modelo de manejo integrado del cultivo, validado para mejorar la sostenibilidad de los Sistemas de Producción de yuca en monocultivo y asocio" a través del convenio No 1828 con la Corporación Colombiana de Investigación Agropecuaria (AGROSAVIA). Además, se agradece a los investigadores Amaury Espitia y Joaquín García del centro de investigación 
Turipana de AGROSAVIA por su participación en el PROYECTO.

Al señor Jaime Luis Herrera Villa por su colaboración en el trabajo de campo.

\section{REFERENCIAS}

Aldrich, R. y Kremer, R. 1997. Competitiveness of weeds. In: Aldrich, R. J.; Kremer, R. J. Principles in weed management. Ames: lowa State University Press/Ames p 1534.

Arismendi, L. 2001. Investigación sobre el cultivo de la yuca (Manihot esculenta Cranz.) en el Oriente de Venezuela. UDO Agricola 1(1): 1-10.

Aristizabal, J., Sánchez, T. y Mejía, D. 2007. Guía técnica para producción y análisis de almidón de yuca. Boletín de Servicios Agrícolas de la FAO 63. Roma. 129 p. http://agris.fao.org/agris-search/search. do? recordID=XF2016011134

Báez, J., Antequera, R., Ramos, J., Gutiérrez, W. y Medrano, C. 1998. Densidad de siembra y control de malezas en el cultivo de la yuca (Manihot esculenta Crantz) en siembra directa bajo las condiciones de la planicie de Maracaibo. Facultad de Agronomía (LUZ) 15 (5): 429-438.

Blanco, Y., Leyva, Á. y Castro, I. 2014. Determinación del periodo crítico de competencia de arvenses en el cultivo de maíz (Zea mays, L.). Cultivos Tropicales, vol. 35, núm. 3, julio-septiembre, 2014, pp. 62-69.

Barreyro, R. y Sánchez, G. 2002. Delimitación del periodo crítico de competencia de malezas en cultivo de lino (Linum usitatissinum). Planta Daninha, 20 (3): 399-403.

Cadavid, L., Acosta, A. y El-Sharkawy, M. 1993. Manejo de un suelo arenoso en Pivijay, Magdalena dedicado a la producción de yuca (Manihot esculenta Crantz). Suelos Ecuatoriales (Colombia) 23(1-2):155161.
Calle, F. 2002. Control de malezas en el cultivo de la yuca. En: La yuca en el tercer milenio: sistemas modernos de producción, procesamiento, utilización y comercialización. pp. $126-128$. Compilado por: B. Ospina y H. Ceballos. Publicación CIAT; Nº 327. Cali, Colombia.

Caraballo, L., y Velásquez, E. 2000. Respuesta de tres cultivares de yuca a diferentes condiciones hídricas y fechas de cosecha. Agronomía Tropical, 50 (2): 267-284.

Ceballos, H., Morantes, N., Calles, F., Lenis, J., Jaramillo, G. y Pérez, J. 2002. Mejoramiento genético de la yuca. En: Ospina B, Ceballos H (Comps). La Yuca en el Tercer Milenio. CIAT. Cali, Colombia, $p$ 295-325.

Centro Internacional de Agricultura Tropical, CIAT, 1981. Manejo y Control de malezas en el cultivo de yuca. Serie 043w.03.01. Segunda Edición, Calí, Colombia.

Girón, C. y Alfonso, E. 2000. Manejo integrado de malezas en yuca. Agronomía Tropical, 50 (1): 31-40.

Gutiérrez, W., Morán, J., Daboin, B., Ferrer, J., Medina, B. y Villalobos, Y. 2008. Evaluación de diferentes herbicidas sobre el control de malezas, desarrollo, rendimiento y beneficio neto relativo del cultivo de la yuca Manihot esculenta Crantz bajo las condiciones agroecológicas de la planicie de Maracaibo. Facultad de Agronomía (LUZ) 25 (1): 26-42.

Hruska, A. 1987. Weedy groundcover increases damage to cassava by white grubs in Costa Rica. Tropical Agriculture, 64 (3): 212-216.

Instituto Dominicano de Investigaciones Agropecuarias y Forestales, IDIAF. 2001. Efecto de diferentes métodos de control de malezas en el cultivo de yuca (Manihot esculenta, Crantz), San Juan de la Maguana, Rep. Dom. 
Instituto Nacional de Innovación Agraria. 2014. Labores culturales en el cultivo de yuca. Estación Experimental Agraria San Roque Iquitos. Hoja divulgativa $\mathrm{N}^{0} 17$. http://repositorio.inia.gob.pe/bitstream/ inia/232/1/HD-17-2014-Cultivo de yuca.pdf

Marín, A., Perdomo, D., Albarrán, J., Fuyenmayor, F. y Zambrano, C. 2008. Evaluación agronómica, morfológica y bioquímica de clones élites de yuca a partir de vitroplantas. Interciencia, 33 (5): 365-371.

Mejía de Tafur, M.S. 1994. Fisiología de la yuca (Manihot esculenta Crantz).Cap 3. En B. Ospina y $\mathrm{H}$. Ceballos. La yuca del tercer milenio (p 34- 45). Cali, Colombia.

Paredes, E. 2014. Determinación del período crítico de control de maleza en yuca (Manihot esculenta crantz.) sembrada a dos densidades, en la Provincia de Orellana.Carrera de Ingeniería Agropecuaria. Universidad de las Fuerzas Armadas ESPE. Sede Santo Domingo.

Pérez, A. y Ruiz, K. 2010. Evaluación de un sistema de cinco policultivos (maíz dulce, habichuela, lechuga, rábano y yuca) en el uso equivalente de terreno, ingresos, y control de malezas en Zamorano, Honduras. Proyecto especial de graduación de Ingeniería Agronomica. Escuela Agrícola Panamericana, Zamorano. Honduras. 31 p.
Quiñónez, V. y Moreno, N. 1995. Control de malezas en yuca en Barinas, Venezuela. Agronomía Tropical, 45 (1):85-94.

Radosevich, S., Holt, J. y Ghersa, C. 1997. Associations of weeds and crops. In: Weed ecology implications for management. Inc. NY: John WIley \& Sons. p 201-209.

\section{Rojas, R., Gutiérrez, W., Esparza, D., Medina,} B., Villalobos, Y. y Morales, L. 2007. Efecto de la densidad de plantación sobre el desarrollo y rendimiento del cultivo de la yuca Manihot esculenta Crantz, bajo las condiciones agroecológicas de la Altiplanicie de Maracaibo. Facultad de Agronomía (LUZ), 24: 94-112.

SAS, 2011. Institute. SAS/STAT 9.3 user's guide. SAS Institute. 